\title{
The use of the transabdominal approach to repair Morgagni hernia in a 28-year-old symptomatic female: a case report
}

\author{
Marah Mansour ${ }^{1}$, Ammar Ismail ${ }^{2}$, Maria Alfathi $^{3}$, Tamim Alsuliman $^{4}$, and Adnan Ismail ${ }^{5}$ \\ ${ }^{1}$ Faculty of Medicine, Tartous University \\ ${ }^{2}$ Faculty of Medicine, Tartous University \\ ${ }^{3}$ Damascus University Faculty of Medicine \\ ${ }^{4}$ Saint-Antoine Hospital, AP-HP, Sorbonne University \\ ${ }^{5}$ Kalamoon University Hospital
}

December 7, 2021

\begin{abstract}
Morgagni's hernia is a congenital diaphragmatic hernia, which represents only $3 \%$ of all diaphragmatic hernias. Herein, we report a case of a 28-years old symptomatic female with Morgagni's hernia who underwent a trans-abdominal surgery using a mesh placed.
\end{abstract}

The use of the transabdominal approach to repair Morgagni hernia in a 28-year-old symptomatic female: a case report

The use of the transabdominal approach to repair Morgagni hernia in a 28-year-old symptomatic female: a case report

\section{ABSTRACT}

Morgagni's hernia is a congenital diaphragmatic hernia, which represents only $3 \%$ of all diaphragmatic hernias. Herein, we report a case of a 28-years old symptomatic female with Morgagni's hernia who underwent a trans-abdominal surgery using a mesh placed.

\section{KEY CLINICAL MESSAGE}

Morgagni's hernia is rare hernia and it could be asymptomatic until adulthood which leads to delayed diagnosis and life-threatening complications. Therefore, early diagnosis and treatment are recommended to avoid serious consequences.

Keywords: Morgagni hernia; transabdominal; diaphragmatic hernia; symptomatic; mesh; transthoracic approach

The use of the transabdominal approach to repair Morgagni hernia in a 28-year-old symptomatic female: a case report

\section{INTRODUCTION}

Morgagni's hernia (MH) is the rarest type of congenital diaphragmatic hernias (DHs), representing only $3 \%$ of all DHs [1]. It occurs inside the Morgagni foramen, which is also known as the sternocostal triangle and is located in a triangular space in the anterior aspect of the thoracic cavity [2]. Generally, $\mathrm{MH}$ is found as a sac within the abdominal viscera $(\mathrm{AV})$ on the right side of the sternum in the thoracic cavity. Both left-sided 
and bilateral forms are uncommon [3]. In some cases, it could be presented with respiratory symptoms like frequent chest infections, which are common in children, or gastrointestinal (GI) symptoms such as bowel obstruction [5]. However, in the majority of cases, MH stays asymptomatic until adulthood, which leads to a delayed diagnosis and discovery by coincidence during a non-related workup [3]. Many studies showed that MH could be associated with different congenital defects and several syndromes: Down's syndrome, Noonan's syndrome, Turner's syndrome, and many others [4]. Surgery can be performed as an effective therapy by either transthoracic approach or trans-abdominal (TA) for both symptomatic and asymptomatic patients [3].

\section{CASE PRESENTATION}

A 28-year-old female presented to the Thoracic Surgery Department with dyspnea, palpitation, and a sense of heaviness in the chest with no abdominal symptoms and only a medical history of two cesarean sections. However, the symptoms persisted and worsened for years, especially (pregnancies, lying supine). Physical examination, laboratory findings, and electrocardiogram were within normal limits. The chest X-ray demonstrated a suspected loop of the bowel on the right side of the thorax cavity (Fig.1). A computed tomography (CT) of the chest and abdomen revealed a $\mathrm{DH}$ in the right hemithorax which contained the transverse colon, loops of small bowel and omentum in the hernia sac (HS) but no liver/stomach (Fig.2). Surgery was performed by open laparotomy and the defect measured approximately $10-15 \mathrm{~cm}$ in size. The bowel loops and rest of the HS contents could be pulled easily from the chest back to the abdominal cavity through the defect; only minor adhesions between the bowel loops were present and were lysed. The large hernia orifice was repaired, and a mesh was placed and attached with sutures to the edges of the defect for further support (Fig.3, Fig.4). The HS was not resected and was left in the chest cavity. No complications occurred during the surgery. Post-operation, the patient recovered well and was discharged six days after surgery. One month of follow-up, physical examination was unremarkable, and the chest X-ray showed no hernia recurrence or complication related to the HS (Fig.5).

\section{DISCUSSION AND CONCLUSION}

Giovanni Morgagni was the first one who described this DHs in $1769[3,6]$. Although the defect is present since birth, an MH may remain asymptomatic until adulthood or present with relatively mild and nonspecific symptoms, and can often be discovered incidentally on a routine X-ray [4, 7]. It begins small to be expanded over time, allowing the AV to herniate into the thorax cavity. The HS is present in $90 \%$ of cases and it most often contains omentum and parts of the transverse colon, other abdominal organs can also be found like the stomach and parts of the small bowel/the liver [8]. Commonly, it presents with GI symptoms (bloating, fullness, discomfort in the abdomen or subcostally) or respiratory symptoms (dyspnea and chest discomfort) [3]. Herein, the patient presented with chest discomfort and other respiratory symptoms. No significant abdominal symptoms were present. The MH diagnosis is based on chest X-rays, CT scans, and GI tract contrast radiography [9]. On a typical chest X-ray the hernia presents as a round shadow in the cardiophrenic angle, a lateral film may reveal its location anterior close to the anterior chest wall and the diaphragm. This shadow may appear to be opaque and homogenous if the hernia contains solid contents like the omentum, but the air could be visible in the mass in case it contains parts of the bowel, mostly the transverse colon [3]. Using a CT scan can assist in further investigating any doubted mediastinal mass on $\mathrm{X}$-ray, and thereby can be considered a reliable tool in the preoperative workup of an $\mathrm{MH}$ [6]. The CT scan of $\mathrm{MH}$ could be variable according to the HS content. However, it may be characterized as a pericardial fat density with linear densities which are consistent with omental blood vessels, an abnormally high position of the transverse colon, or visible bowel loops within the chest [6]. An empty HS or a sac with solid contents may complicate or delay the diagnosis [9]. A differential diagnosis includes a lipoma, pleuropericardial cyst, thymoma, diaphragmatic cyst, or a pericardial fat pad [7]. If parts of the bowel are present in the HS, a radiographic study with a barium enema and MRI can be very useful in confirming the diagnosis [3, 9]. A chest X-ray showed an air-containing mass in the right cardiophrenic angle adjacent to the heart in our case (Fig.1). A CT scan showed the presence of bowel loops in the chest (Fig.2). Surgery is the definitive treatment and recommended for both symptomatic and asymptomatic cases to avoid complications like 
volvulus or strangulation. Moreover, there is still some controversy about the preferred surgical technique. Some prefer the TA, while others advocate the transthoracic, laparoscopic, or thoracoscopic approach [4]. The TA is usually preferred for easier hernia reduction. The bowel loops or AV can be pulled down into the abdominal cavity. The HS can be resected [5]. Although the laparoscopic approach has shown favorable results, it is not appropriate for cases that are considered surgical, like strangulation [8]. In general, small hernias can be repaired by direct suturing, whereas large defects usually require mesh repair [4]. Here, the TA was preferred, and a mesh was used to repair the defect (Fig.3, Fig.4). In conclusion, this publication aims to demonstrate the challenges of diagnosing and managing Morgagni's hernia in adults.

\section{REFERENCES}

1. Chin EF, Duchesne ER. The parasternal defect. Thorax. 1955 Sep;10(3):214.

2. Paris F, Tarazona V, Casillas M, Blasco E, Canto A, Pastor J, Acosta A. Hernia of morgagni. Thorax. 1973 Sep 1;28(5):631-6.

3. Comer TP, Clagett OT. Surgical treatment of hernia of the foramen of Morgagni. The Journal of thoracic and cardiovascular surgery. 1966 Oct 1;52(4):461-8.

4. Gedik E, Tuncer MC, Onat S, Avcl A, Tacylldlz I, Bac B. A review of Morgagni and Bochdalek hernias in adults. Folia Morphologica. 2011;70(1):5-12.

5. Loong TP, Kocher HM. Clinical presentation and operative repair of hernia of Morgagni. Postgraduate medical journal. 2005 Jan 1;81(951):41-4.

6. Minneci PC, Deans KJ, Kim P, Mathisen DJ. Foramen of Morgagni hernia: changes in diagnosis and treatment. The Annals of thoracic surgery. 2004 Jun 1;77(6):1956-9.

7. Kiliç D, Nadir A, Döner E, Kavukçu Ş, Akal M, Özdemir N, Akay H, Ökten I. Transthoracic approach in surgical management of Morgagni hernia. European journal of cardio-thoracic surgery. 2001 Nov 1;20(5):10169.

8. Karamustafaoglu YA, Kuzucuoglu M, Tarladacalisir T, Yoruk Y. Transabdominal subcostal approach in surgical management of Morgagni hernia. European journal of cardio-thoracic surgery. 2011 Jun 1;39(6):100911.

9. Karamustafaoglu YA, Kuzucuoglu M, Tarladacalisir T, Yoruk Y. Transabdominal subcostal approach in surgical management of Morgagni hernia. European journal of cardio-thoracic surgery. 2011 Jun 1;39(6):100911.

Figure legends

Fig.1: A chest X-ray image showing a suspected loop of bowel on the right side of the chest.

Fig.2: An axial CT scan showing the herniated bowel on the right side of the chest.

Fig.3: A trans-abdominal surgery showing the orifice of the Morgagni hernia.

Fig.4: Intraoperative image showing the mesh that was used for repairing the defect.

Fig.5: One month of follow-up, a chest X-ray image confirming that there is no hernia recurrence. 

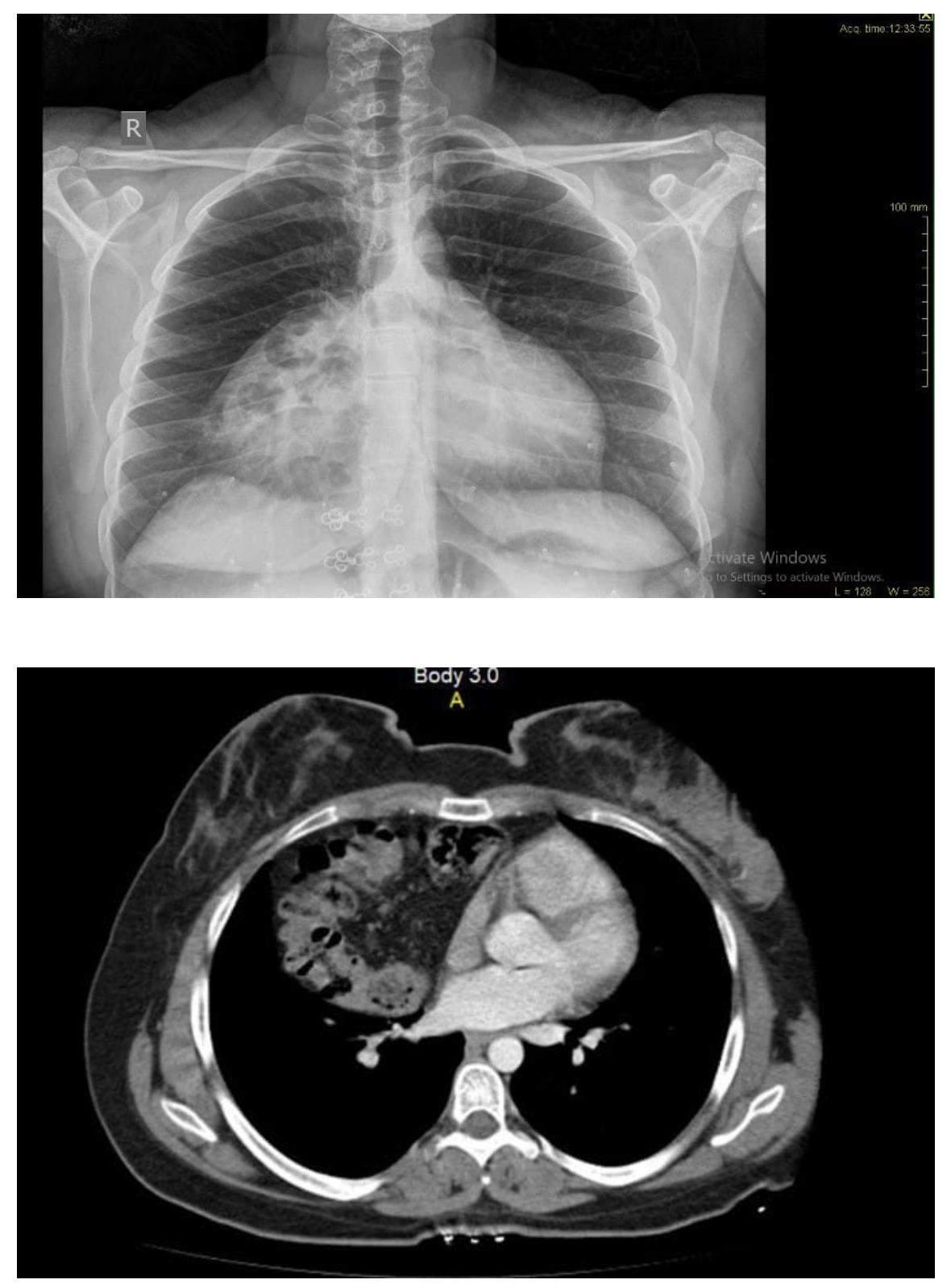


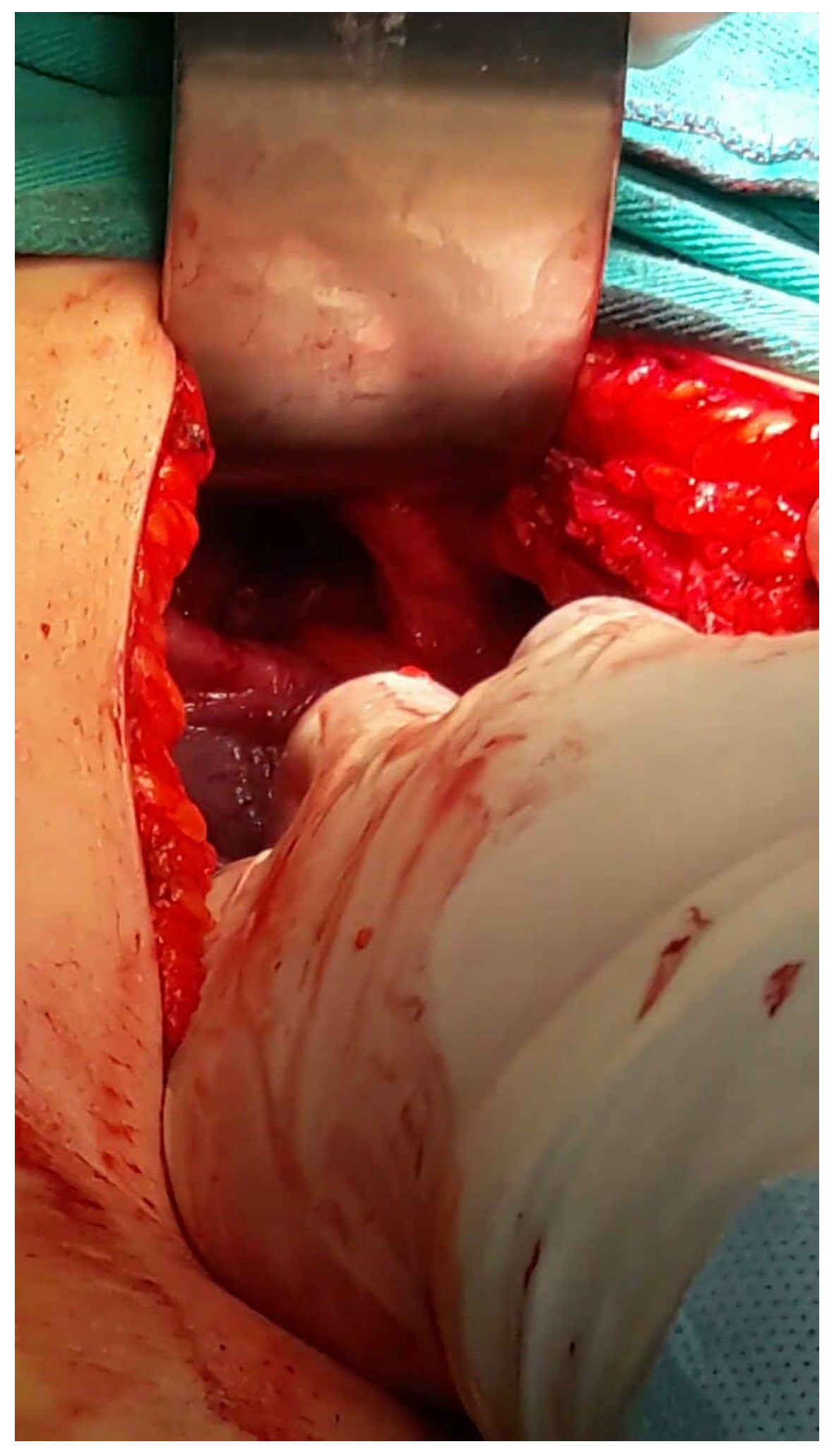



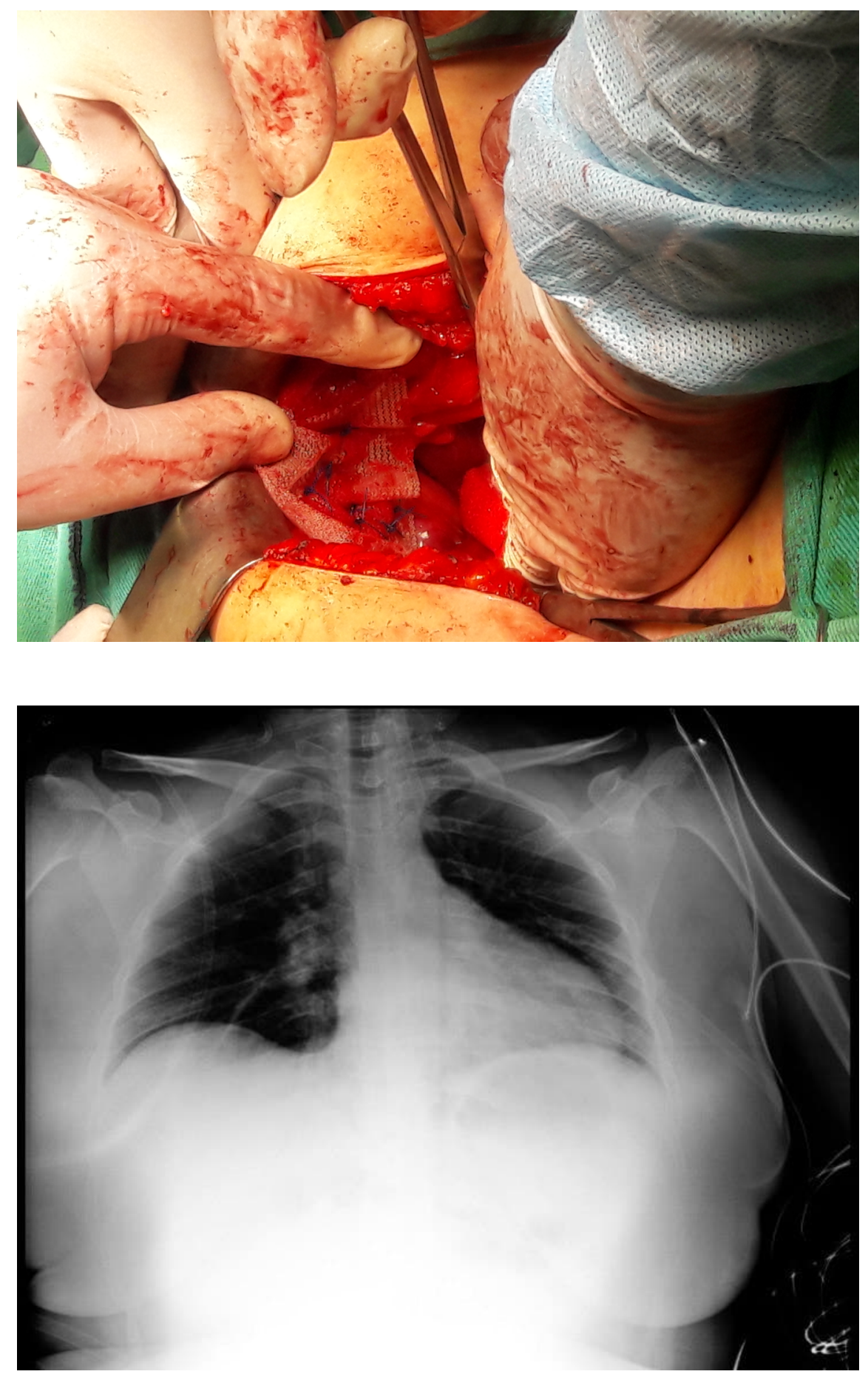